\title{
Analysis of two-dimensional photoelectron momentum spectra and the effect of the long-range Coulomb potential in single ionization of atoms by intense lasers
}

\author{
Zhangjin Chen, ${ }^{1}$ Toru Morishita, ${ }^{1,2}$ Anh-Thu Le, ${ }^{1}$ M. Wickenhauser, ${ }^{3}$ X. M. Tong, ${ }^{4}$ and C. D. Lin ${ }^{1}$ \\ ${ }^{1}$ J. R. Macdonald Laboratory, Physics Department, Kansas State University, Manhattan, Kansas 66506-2604, USA \\ ${ }^{2}$ Department of Applied Physics and Chemistry, The University of Electro-Communications, \\ 1-5-1 Chofu-ga-oka, Chofu-shi, Tokyo 182-8585, Japan \\ ${ }^{3}$ Institute for Theoretical Physics, Vienna University of Technology, A-1040 Vienna, Austria \\ ${ }^{4}$ Institute of Materials Science, Graduate School of Pure and Applied Science, and Center for Computational Sciences, \\ University of Tsukuba, 1-1-1 Tennodai, Tsukuba, Ibaraki 305-8577, Japan
}

(Received 26 September 2006; published 6 November 2006)

\begin{abstract}
Two-dimensional (2D) electron momentum distributions and energy spectra for multiphoton ionization of atoms by intense laser pulses, calculated by solving the time-dependent Schrödinger equation (TDSE) for different wavelengths and intensities, are compared to those predicted by the strong-field approximation (SFA). It is shown that the momentum spectra at low energies between the TDSE and SFA are quite different and the differences arise largely from the absence of a long-range Coulomb interaction in the SFA. We further found that the low-energy 2D momentum spectra from the TDSE exhibit ubiquitous fanlike features where the number of stripes is due to a single dominant angular momentum of the low-energy electron. The specific dominant angular momentum in turn has been found to be decided by the minimum number of photons needed to ionize the atom only. The electron momentum spectra predicted by models modified from the SFA are also examined and found to lack the fanlike features as in the SFA.
\end{abstract}

DOI: 10.1103/PhysRevA.74.053405

PACS number(s): $32.80 . \mathrm{Rm}, 32.80 . \mathrm{Fb}, 42.50 . \mathrm{Hz}$

\section{INTRODUCTION}

In recent years the phenomenon of above-threshold ionization (ATI) has been extensively studied with femtosecond laser pulses. In this process an atom absorbs more photons than the minimum number required for the ejection of an electron. By measuring the energy of the electron, the characteristic ATI spectra are peaks separated by the photon energy, while their positions are shifted by the ponderomotive potential. For pulses of durations of the order of 10-20 fs, calculations have shown that the pronounced ATI peaks are often accompanied by subpeaks. These subpeaks have been attributed to the rapidly changing ponderomotive potential in the short laser pulse [1-3].

Experimentally more detailed information on ATI electrons can be determined by measuring the angular distributions. Such experiments have been carried out for longer pulses at lower intensities and many features in the angular distributions have been attributed to contributions from the so-called Freeman resonances [4]. Using the multiphoton ionization picture, Freeman resonances are understood to come from atomic Rydberg states that are ac-Stark shifted into resonance with the absorption of multiple photons. Examples of such careful studies together with a theoretical analysis on Ar have been reported by Wiehle et al. [5], for example, using lasers of durations of the order of $100 \mathrm{fs}$. For shorter pulses, on the order of $30 \mathrm{fs}$, two-dimensional (2D) electron momentum spectra have been measured using the COLTRIMS apparatus, with lasers of much higher intensities, using typical 800-nm Ti-sapphire lasers [6], and most recently, with lasers in the 400-800-nm region [7]. These later experiments were carried out with Keldysh parameters less than 1, and thus are considered in the tunneling ionization regime. In the tunneling ionization model, the electron spectra are expected to be featureless. Instead, earlier data from Rudenko et al. [6] showed striking features in their 2D electron momentum spectra, as well as in the momentum spectra projected onto the direction of the laser polarization. Similar features in the 2D electron momentum spectra have been seen in the data of Maharjan et al. [7] for 400-800-nm wavelengths.

Theoretically the ATI electron spectra of atoms can be calculated in principle by solving the time-dependent Schrödinger equation (TDSE) directly [5,8-12]. While there exist many computational packages in the literature, few calculations on the electron angular distributions or the 2D electron momentum distributions have been reported. Since the experiment of Rudenko et al. [6], various theoretical models have been proposed to interpret these experimental data $[2,13,14]$. One major difficulty associated with all these theories is that they have not examined the laser intensity dependence extensively. In the tunneling ionization region, the electron spectra, including most of the distinct features, change rapidly with the laser intensity. For a highly focused laser pulse, contributions to the electron yields from the interaction volume of varying laser intensities should be included. This volume integration has the tendency to smooth out most distinct features in the spectra unless the features are relatively independent of the laser intensity.

We have carried out extensive calculations on the 2D electron spectra of various atoms by lasers of wavelengths from $400 \mathrm{~nm}$ to $800 \mathrm{~nm}$ by directly solving the TDSE in a laser field, within the single-electron approximation for multielectron targets. We have also performed calculations based on the strong-field approximation (SFA) [15-17]. In a previous paper [1] we emphasized the qualitative similarity between the two theories, especially in the electron energy spectra if they are normalized to each other. In this paper, we 
address the differences in the two theories, particularly in the low-energy $2 \mathrm{D}$ electron momentum spectra. In the TDSE calculation we found that at low energies the 2D momentum spectra exhibit pronounced discrete stripes fanning out radially from the center. These features are clearly seen in the data of Maharjan et al. [7], in spite of the volume integration. In the SFA calculations, the low-energy features are distinctly different: In general there are fewer stripes and they tend to lie vertically with respect to the laser polarization direction.

To assess the origin of this major difference, we have carried out TDSE calculations by setting the Coulomb tail of the model potential to zero prematurely with a cutoff function. In other words, we perform TDSE calculations for an atom with short-range potential but maintaining nearly identical ionization energy as in a real atom. Interestingly, the low-energy electron momentum spectra from these calculations change drastically and become much similar to the SFA results. We can definitely attribute the low-energy features in the TDSE calculations to the effect of the asymptotic Coulomb potential seen by the electron.

To understand these low-energy features in the $2 \mathrm{D} \mathrm{mo-}$ mentum spectra and how to relate the number of stripes to the laser parameters and target atoms, we have examined a number of atoms with outer $s$ shell and $p$ shell for lasers with different wavelengths and intensities. We analyzed the distributions of the angular momentum compositions of the lowenergy electrons and found that they tend to be dominated by a single angular momentum $L$. We have been able to associate this $L$ with the minimum number of photons required to ionize the atom at a given peak laser intensity. We have made a model where $L$ can be predicted for any laser intensity. Since $L$ does not change until the minimum number of photons to ionize the atom increases by one more unit-i.e., at the beginning of the channel crossing - this implies that the low-energy feature does not change rapidly with the laser intensity. This would qualitatively explain the structures observed by Maharjan et al. [7] and by Rudenko et al. [6].

The rest of this paper is organized as follows. In Sec. II the theoretical methods and the approximations used in the present calculations are presented. The results are given in Sec. III. The last section summarizes our results, accompanied by a discussion of possible further studies. Atomic units $\left(m_{e}=\hbar=e=1\right)$ are used throughout this paper unless stated otherwise.

\section{THEORETICAL MODELS}

In this section, we summarize the various theoretical models used in the calculation of the ATI spectra.

\section{A. Time-dependent Schrödinger equation method}

The time-dependent Schrödinger equation for an electron under the influence of a classical electromagnetic field reads

$$
i \frac{\partial}{\partial t} \Psi(\mathbf{r}, t)=\left[H_{0}+H^{\prime}\right] \Psi(\mathbf{r}, t) .
$$

Here $H_{0}$ is the unperturbed Hamiltonian for the atom and $H^{\prime}$ is the atom-field interaction. For multielectron atoms, one often makes the single-active-electron (SAE) approximation in which it is assumed that the orbitals of all the electrons but one are frozen in the laser field. In the SAE model, the oneelectron Hamiltonian $H_{0}$ is expressed as

$$
H_{0}=-\frac{1}{2} \nabla^{2}+V(\mathbf{r}) \text {. }
$$

In this work, we use the effective atomic potential which is parametrized by [18]

$$
V(\mathbf{r})=-\frac{Z+a_{1} e^{-a_{2} r}+a_{3} r e^{-a_{4} r}+a_{5} e^{-a_{6} r}}{r},
$$

where $Z$ is the charge of the residual ion and the parameters $a_{i}$ are obtained by fitting the numerical potential calculated from the self-interaction free density functional theory [9].

Within the dipole approximation and the length gauge, the atom-field interaction takes the form

$$
H^{\prime}=\mathbf{r} \cdot \mathbf{F}(t) \text {. }
$$

For a linearly polarized laser pulse with the electric field $\mathbf{F}(t)$ along the $z$ axis,

$$
\mathbf{F}(t)=F_{0} \hat{z} a(t) \cos (\omega t+\varphi),
$$

where $\omega$ is the carrier frequency and $\varphi$ the carrier-envelope phase with the envelope function $a(t)$ chosen to be

$$
a(t)=\cos ^{2}\left(\frac{\pi t}{\tau}\right)
$$

for the time interval $(-\tau / 2, \tau / 2)$ and zero elsewhere. The pulse duration, defined as the full width at half maximum (FWHM), is given by $\Gamma=\tau / 2.75$.

In the numerical calculations, $\Psi(\mathbf{r}, t)$ is expanded as

$$
\Psi(\mathbf{r}, t)=\sum_{n l} C_{n l}(t) f_{n l}(r) Y_{l m}(\hat{\mathbf{r}}),
$$

where $f_{n l}(r) Y_{l m}(\hat{\mathbf{r}})$ is the eigenfunction of $H_{0}$ within the box of $r \in\left[0, r_{\max }\right]$, and $f_{n l}(r)$ and $Y_{l m}$ are expanded by the discrete variable representation (DVR) [19-21] basis set associated with Legendre polynomials while $C_{n l}$ is obtained by using the split-operator method [10]:

$$
\begin{aligned}
C_{n l}(t+\Delta t) \simeq & \sum_{n^{\prime} l^{\prime}}\left\{\exp \left(-i H_{0} \Delta t / 2\right) \exp \left[-i H^{\prime}(t+\Delta t / 2) \Delta t\right]\right. \\
& \left.\times \exp \left(-i H_{0} \Delta t / 2\right)\right\}_{n l, n^{\prime} l^{\prime}} C_{n^{\prime} l^{\prime}}(t),
\end{aligned}
$$

where the matrix elements are evaluated efficiently by using DVR quadrature. For the case of a short-pulse and lowenergy electron considered in this paper, $r_{\max }=1000$ is large enough. Note that in Eq. (7), only $m=0$ is taken into account and this is only true if the atom is initially in the $s$ state. However, for the linearly polarized laser pulse, even though the atom is initially in the $p$ state, for example, the contribution to the ionization probability from $m= \pm 1$ is relatively much smaller in comparison to the $m=0$ component.

The angular distribution or electron momentum distribution can be extracted by projecting the final wave function at the end of the pulse onto eigenstates corresponding to elec- 
trons emitted in the parent ion continuum with a given momentum vector $\mathbf{p}$,

$$
\frac{\partial^{2} P}{\partial E \partial \theta}=\left|\left\langle\Phi_{\mathbf{p}}^{-} \mid \Psi(t=\tau / 2)\right\rangle\right|^{2} 2 \pi p \sin \theta,
$$

where $E=p^{2} / 2$ is the kinetic energy of the detached electron and $\theta$ is the angle between $\hat{\mathbf{p}}$ and the $z$ axis. The continuum state $\Phi_{\mathbf{p}}^{-}$satisfies the equation

$$
\left[-\frac{1}{2} \nabla^{2}+V(\mathbf{r})\right] \Phi_{\mathbf{p}}^{-}=E \Phi_{\mathbf{p}}^{-}
$$

and reads in terms of partial waves

$$
\Phi_{\mathbf{p}}^{-}=\sum_{l} \sum_{m=-l}^{l} i^{l} e^{-i \delta_{l}} R_{p}^{l}(r) Y_{l m}^{*}(\hat{\mathbf{p}}) Y_{l m}(\hat{\mathbf{r}}),
$$

where $\delta_{l}$ is the phase shift. Therefore Eq. (9) can be rewritten as

$$
\begin{aligned}
\frac{\partial^{2} P}{\partial E \partial \theta}= & \left|\sum_{l} i^{l} e^{-i \delta_{l}} Y_{l 0}(\hat{\mathbf{p}}) \sum_{n} C_{n l}(\tau / 2)\left\langle R_{p}^{l}(r) \mid f_{n l}(r)\right\rangle\right|^{2} \\
& \times 2 \pi p \sin \theta .
\end{aligned}
$$

By integrating over $\theta$ in Eq. (9), we obtain the electron energy spectra

$$
\frac{\partial P}{\partial E}=\int \frac{\partial^{2} P}{\partial E \partial \theta} d \theta
$$

\section{B. Strong-field approximation}

While direct solution of the Schrödinger equation in a time-dependent laser field has been widely used, the simpler strong-field approximation [15-17] is of interest for analyzing features of the intense laser-atom interaction.

In the SFA model the momentum-dependent ionization amplitude is given by [22]

$$
f(\mathbf{p})=i \int_{-\infty}^{\infty} d t\left\langle\mathbf{p}+\mathbf{A}(t)\left|H^{\prime}(t)\right| \Psi_{0}\right\rangle \exp [-i S(\mathbf{p}, t)],
$$

where

$$
S(\mathbf{p}, t)=\int_{t}^{\infty} d t^{\prime}\left\{\frac{\left[\mathbf{p}+\mathbf{A}\left(t^{\prime}\right)\right]^{2}}{2}+I_{p}\right\},
$$

with $I_{p}$ the binding energy of the initial state and $\mathbf{A}(t)$ the vector potential. The field-free continuum state $|\mathbf{p}+\mathbf{A}(t)\rangle$ takes the form

$$
\langle\mathbf{r} \mid \mathbf{p}+\mathbf{A}(t)\rangle=\frac{1}{(2 \pi)^{3 / 2}} \exp \{i[\mathbf{p}+\mathbf{A}(t)] \cdot \mathbf{r}\} .
$$

The ground state wave function $\Psi_{0}$ is obtained numerically by solving the differential equation

$$
\left[-\frac{1}{2} \nabla^{2}+V(\mathbf{r})\right] \Psi_{0}=E_{0} \Psi_{0},
$$

where $E_{0}=-I_{p}$ is the energy of the ground state.
The SFA model has been widely applied to various problems, including ATI [1] and high-harmonic generation (HHG) [22], and has also been shown to be reliable for loworder above-threshold detachment from negative ions, for which there is no long-range Coulomb interaction $[23,24]$. Despite the success of the SFA, there are still open questions on the role of the long-range Coulomb interaction between the outgoing photoelectron and the residual core. We will address this issue by specific examples.

\section{Modification to the SFA}

The Coulomb correction for the Volkov wave function of the final continuum state was first suggested by Krainov and Shokri [25] for the case of the ground state of atomic hydrogen and then generalized to arbitrary atoms or atomic ions by Krainov [26]. Adopting this correction, the modified SFA (MSFA) ionization amplitude reads

$$
f_{\mathrm{M}}(\mathbf{p})=i \int_{-\infty}^{\infty} d t\left\langle\mathbf{p}+\mathbf{A}(t)\left|I H^{\prime}(t)\right| \Psi_{0}\right\rangle \times \exp [-i S(\mathbf{p}, t)],
$$

where

$$
I=\left[\frac{4 I_{p}}{F_{0} r}\right]^{Z / \sqrt{2 I_{p}}} .
$$

Note that this modified SFA corrects the dipole transition amplitude, with the phase left unchanged. This has been interpreted as to include the tunneling contribution to the ionization step $[25,26]$. Application of the MSFA to $\mathrm{He}^{+}$by Chirilă and Potviege [27] shows that the total ionization probability versus laser intensity agrees quite well in comparison to the results from the tunneling ionization theory. By applying the MSFA to other atoms, however, we found that this agreement does not apply to other atoms in general; see below.

\section{Orthonormalized strong-field approximation}

The SFA can be understood as similar to the first Born approximation theory for rearrangement collisions. Note that the total Hamiltonian equation (1) can be partitioned in at least two different ways:

$$
H=\left[-\frac{1}{2} \nabla^{2}+V(r)\right]+H^{\prime}=\left[-\frac{1}{2} \nabla^{2}+H^{\prime}\right]+V(r) .
$$

The ground state is the eigenstate of $\left[-\nabla^{2} / 2+V(r)\right]$, while the Volkov state is the eigenstate of $\left[-\nabla^{2} / 2+H^{\prime}\right]$. The SFA is the "prior" form of the first Born approximation in which the initial and final states are not orthogonal. A similar first Born theory appears in high-energy charge transfer theory [28]. In charge transfer theory, the initial state is the eigenstate of the target atom and the final state is the eigenstate of the projectile atom; the first Born approximation is called Oppenheimer-Brinkman-Kramers (OBK) theory [29,30]. Interestingly, OBK theory is known to always overestimate the 
actual charge transfer cross sections, even though it predicts the correct energy dependence. In this respect it is analogous to the SFA, which is known to give ionization probabilities that are too small, but once normalized it does give nearly the correct intensity dependence.

In charge transfer theory one can improve the OBK theory by forcing the final state to be orthonormal to the initial state [31]. We have extended a similar procedure to the SFA, and the resulting modified SFA is called orthonormalized strongfield approximation (OSFA) here. The OSFA ionization amplitude is given by

$$
f_{\mathrm{O}}(\mathbf{p})=i \int_{-\infty}^{\infty} d t \frac{\left\langle\mathbf{p}+\mathbf{A}(t)\left|H^{\prime}(t)\right| \Psi_{0}\right\rangle}{1-|O(t)|^{2}} \exp [-i S(\mathbf{p}, t)]
$$

where

$$
O(t)=\left\langle\mathbf{p}+\mathbf{A}(t) \mid \Psi_{0}\right\rangle .
$$

Here we have neglected the phase factor of $e^{-i \delta(t)}$ which represents the distortion of the wave function near the nucleus. Interestingly, the total ionization probabilities calculated from the OSFA for atomic hydrogen has been found to be in good agreement with the TDSE calculations. The electron spectra as well as the 2D momentum spectra calculated from OSFA, however, are closer to the SFA. In other words, it only improves the total ionization probability. Additional results will be discussed in the next section.

\section{RESULTS AND DISCUSSION}

\section{A. Modeling the cutoff of the long-range Coulomb potential}

To examine the role of the long-range Coulomb potential on the ATI spectra, we introduce a cutoff function to modify $V(r)$ such that it approaches zero much earlier, similar to a short-range potential. Thus we choose

$$
\tilde{V}\left(r, r_{c}\right)=\left\{\begin{array}{cc}
V(r), & r<r_{c}, \\
V(r)-\tanh \left[\left(r-r_{c}\right) / 10\right] / r, & r \geqslant r_{c},
\end{array}\right.
$$

and let $r_{c}=2$ and 5, respectively. In Fig. 1 we show $r V(r)$ vs $r$ for an argon atom and the two modified potentials. We make sure that the binding energy is not much affected by the change of the potential at large $r$. The " $3 p$ " binding energies calculated from the two modified potentials are 0.57395 and 0.57937 for $r_{c}=2$ and 5, respectively, in comparison to 0.57938 for the real Ar atom.

The effect of the long-range Coulomb potential on the ATI spectra has also been investigated by comparing the energy spectra predicted by the SFA with those obtained via numerical solution of the TDSE [32-34]. However, Bauer et al. $[32,33]$ set the potential to vanish suddenly at a given $r_{c}$. This introduces a discontinuity in the potential which would introduce undesirable reflection into the continuum wave function and thus the electron spectra.

\section{B. Effect of the Coulomb potential on the electron momentum spectra}

In this section we compare the two-dimensional electron momentum spectra for two different laser pulses calculated

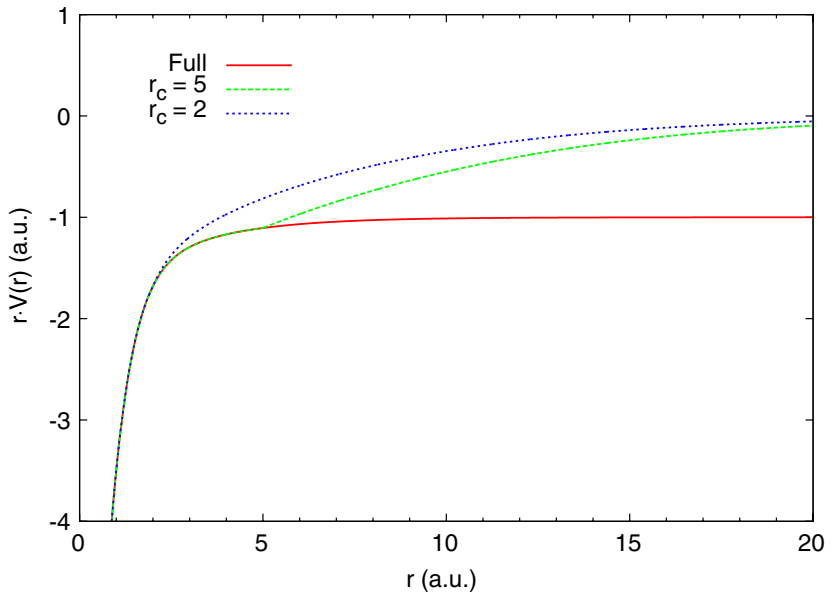

FIG. 1. (Color online) R-weighted potential, or effective charge, of argon, together with models with potentials cut off at different $r_{c}$ (see text).

using the following theoretical models: (a) solving the TDSE using the actual model potential for Ar, (b) solving the TDSE using the modified potential with cutoff radius $r_{c}=5$, (c) solving the TDSE using the modified potential with cutoff radius $r_{c}=2$, and (d) the SFA. To characterize a laser pulse, we also use the Keldysh parameter, defined by $\gamma$ $=\sqrt{I_{p} /\left(2 U_{p}\right)}$, where $U_{p}$ is the ponderomotive potential. Since the electron is ionized in the laser field, the minimum number of photons $N$ needed to ionize the atom is

$$
0<N \hbar \omega-I_{p}-U_{p}<\hbar \omega .
$$

For the first calculation the laser pulse has a wavelength of $400 \mathrm{~nm}$, peak intensity of $3.2 \times 10^{14} \mathrm{~W} / \mathrm{cm}^{2}$, and pulse duration (FWHM) of 10 fs. For this laser the Keldysh parameter is 1.28 and it takes at least seven photons to ionize Ar. In the second calculation the laser has a wavelength of $600 \mathrm{~nm}$, peak intensity of $1.4 \times 10^{14} \mathrm{~W} / \mathrm{cm}^{2}$, and pulse duration of $10 \mathrm{fs}$. It has nearly the same Keldysh parameter of 1.29, and it takes at least ten photons to ionize Ar.

We first mention that the total ionization probabilities for the 400-nm laser for the four different models are 0.531, $0.566,0.340$, and 0.123 , respectively. If one uses the ADK theory, with the parameters from Tong et al. [35], the ionization probability is 0.723 . For the $600-\mathrm{nm}$ pulse, the total ionization probabilities for the four calculations are 0.031, $0.024,0.0088$, and 0.0015 , respectively, while the ADK theory predicts 0.014 . In both cases, the probability predicted from the SFA is the worst, ranging from a factor of 4 too small in the 400-nm case to a factor of about 20 in the 600 -nm case. For the ADK theory, the error is within $40 \%$ for the 400-nm case and within a factor of 2 for the 600 -nm case.

In Fig. 2 we show the 2D electron momentum spectra for the $400-\mathrm{nm}$ and in Fig. 3 for the $600-\mathrm{nm}$ laser pulses. We will take case (a) in each figure as the "exact" results. At first glance, the 2D momentum spectra appear to be fairly similar among the four different calculations, in terms of the ring structure and the number of peaks within each ring. A closer look reveals that there are some differences. In Fig. 2(a), 


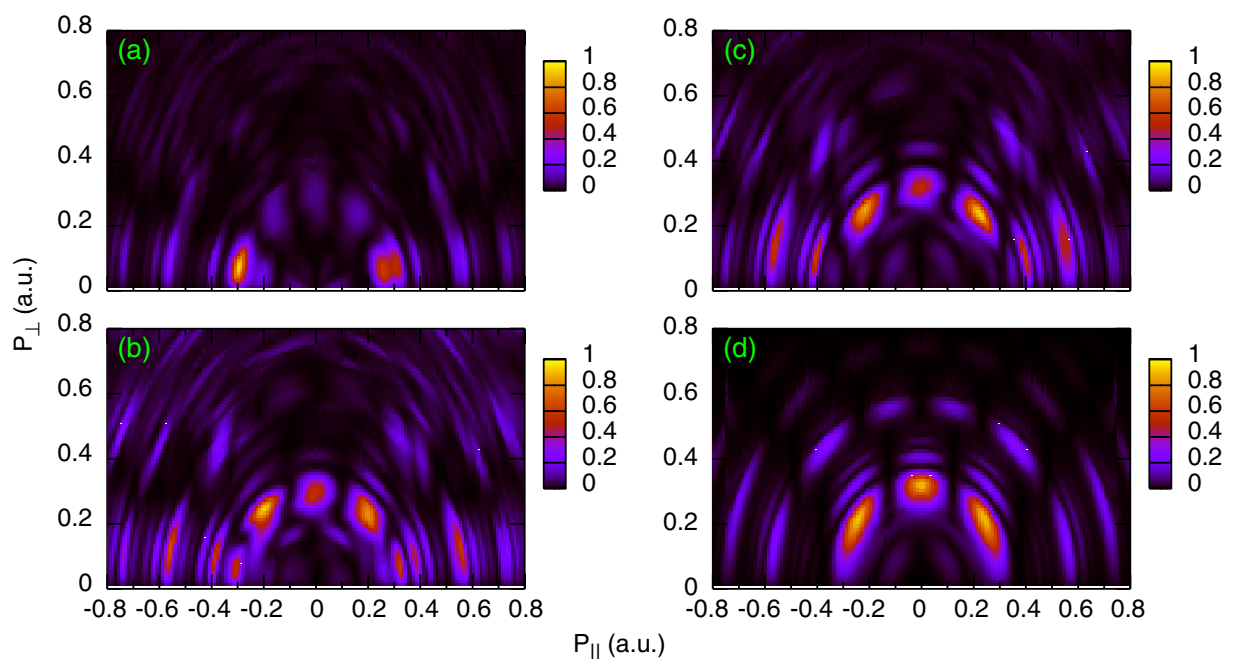

FIG. 2. (Color online) Photoelectron 2D momentum distributions for single ionization of argon by a 10-fs (FWHM) laser pulse with wavelength of $400 \mathrm{~nm}$ at the peak intensity of $3.2 \times 10^{14} \mathrm{~W} / \mathrm{cm}^{2}$, calculated by the (a) full TDSE, (b) TDSE with $r_{c}=5$, (c) TDSE with $r_{c}=2$, and (d) SFA.

within the first ring, the intensity tends to be much higher for the peaks that are closer to the axis-i.e., closer to the polarization direction. The change from Fig. 2(a) to Fig. 2(b) is not small even though only the Coulomb tail beyond $r=5$ has been modified. In other words, we see a substantial change in the $2 \mathrm{D}$ momentum distributions due to the long-range Coulomb potential; i.e., the Coulomb potential in the large- $r$ region can make significant modifications to the angular distribution of the ATI electrons. Comparing Figs. 2(b) and 2(c), the change is less severe. We thus conclude that the modification to the spectra from Fig. 2(a) is mostly due to the Coulomb potential beyond 5 a.u.

In the case of SFA, the full potential $V(r)$ is not included once the electron is ionized. Figure 2(d) appears to be closer to Figs. 2(b) and 2(c). We thus conclude that the difference in the 2D momentum spectra between the TDSE and SFA is due to the long-range Coulomb potential. However, the ring structure, even the number of peaks (or stripes) within the ring, is more or less the same.

The 2D spectra for the $600-\mathrm{nm}$ pulse in Fig. 3 reveal similar trends in the four different calculations, in terms of the ring structures and the tendency of increasing intensity in the large-angle region (near $90^{\circ}$ ), from Fig. 3(a) to Fig. 3(d). In this case, there are additional pronounced features in the low-energy part of the spectra. In Fig. 3(a), the spectra show six stripes of electrons fanning out radially from the center. In Fig. 3(b), the stripes can still be seen, but the number appears to have decreased. This trend is more clear in Figs. 3(c) and 3(d) where the number has decreased to 4. Furthermore, the stripes are not radial, but rather perpendicular to the polarization axis. As shown by Wickenhauser et al. [2], vertical stripes of the 2D spectra are expected within the SFA theory. We thus witnessed another effect of the long-range Coulomb potential on the low-energy electron ATI spectra: it changes from the vertical stripes predicted by the SFA to the radial stripes fanning out from the center when the full Coulomb potential is included.

The stripes in Fig. 3(a) appear to be a general feature of the 2D electron momentum distributions from the full TDSE solutions. Although it is not seen in Fig. 2(a), a blowup of the low-energy spectra, as shown in Fig. 4(a), reveals the same features. In this case, Fig. 4(a) shows four well-defined stripes, while in Fig. 4(b), they are irregular stripes, and gradually they change to two stripes in Figs. 4(c) and 4(d). In this case the stripes become nearly vertical, separated by the nodal line at $90^{\circ}$. We note that the parity in the $2 \mathrm{D}$ spectra is the same in all four calculations.

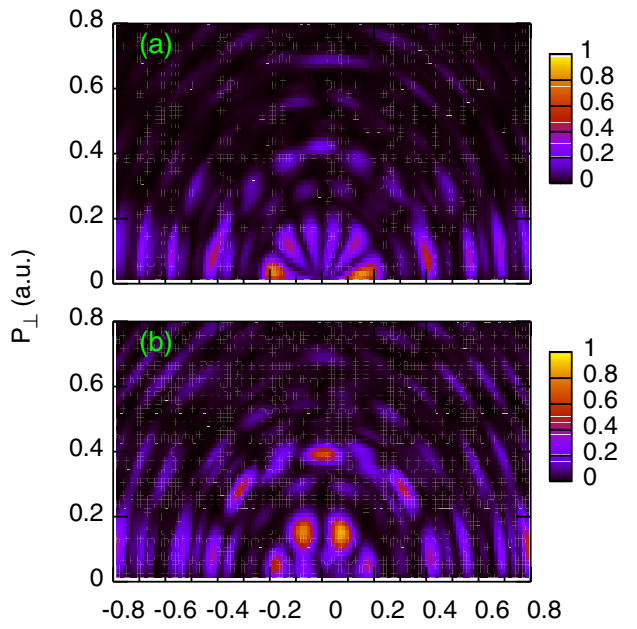

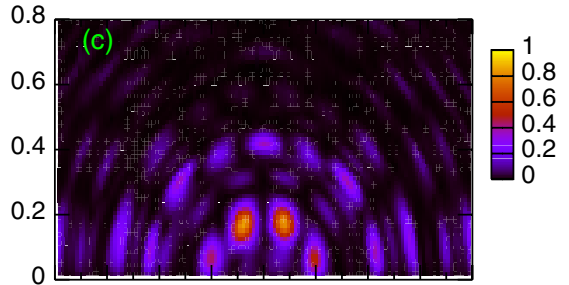

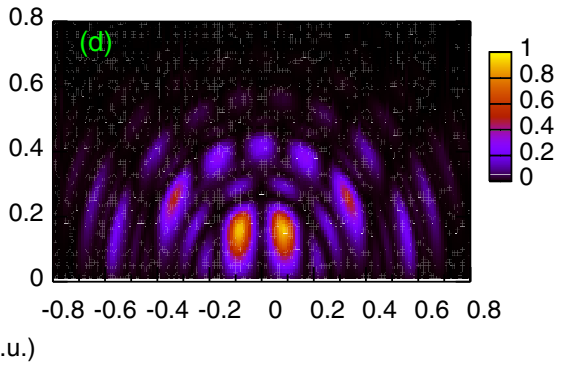

FIG. 3. (Color online) Photoelectron 2D momentum distributions for single ionization of argon by a 10-fs (FWHM) laser pulse with wavelength of $600 \mathrm{~nm}$ at the peak intensity of $1.4 \times 10^{14} \mathrm{~W} / \mathrm{cm}^{2}$, calculated by the (a) full TDSE, (b) TDSE with $r_{c}=5$, (c) TDSE with $r_{c}=2$, and (d) SFA. 


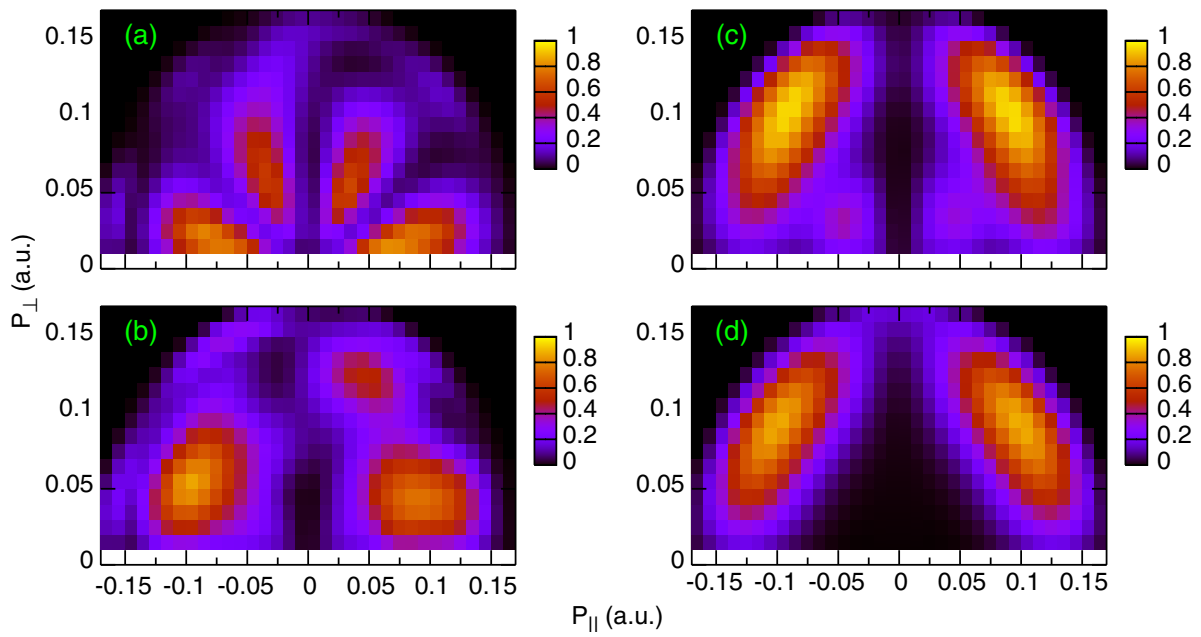

FIG. 4. (Color online) Same as Fig. 2 but for the low-energy region.

\section{Low-energy electron energy spectra}

In Fig. 5(a) we show the electron energy spectra calculated with the four different methods using a 400-nm laser pulse. In this case the total probabilities from these models differ by only within a factor of 4 . The ATI spectra from all four calculations show dominant peaks separated by the photon energy, but with the presence of subpeaks. The relative strength of the subpeaks with respect to the main peaks is expected to decrease as the pulse duration is increased [1]. The spectra from the "exact" calculation tend to shift to the lower-electron-energy side, which can be explained qualitatively as due to "Coulomb focusing"; i.e., the electrons are slowed down due to the pull of the Coulomb attraction from the ionic core. In Fig. 5(b), the electron spectra are normalized at the first ATI peak. This figure shows that once normalized, the electron spectra appear to be rather close to the exact calculation, including the simple SFA model. Since these peaks shift rapidly with changing laser intensity, the energy shift from these theoretical models may not be seen experimentally when the finite size of the gas volume is taken into account. We thus believe that it is difficult to tell the difference of these models from the measured electron energy spectra.

\section{Coulomb focusing and transverse electron momentum spectra}

The transverse electron momentum spectra of $\mathrm{Ar}$ and a few other atoms have been measured experimentally $[36,37]$. By comparing with the prediction of the SFA, the narrower distribution of the experimental data has been attributed to the effect of Coulomb focusing [37] -i.e., the effect of electrons being pulled toward the laser polarization direction by the attractive Coulomb force once it is ionized.

Using the 400-nm data as an example, we have calculated the transverse electron momentum distributions of the ejected electrons. In Fig. 6(a) we show the actual electron yield from the calculations using the four different models. Clearly the exact calculation exhibits a much sharper cusplike distribution, while for successive models the distributions become broader. Note that the change occurs mostly in the $p_{\perp}=0$ region; i.e., Coulomb focusing is more significant only in the low-perpendicular-momentum region. If the dis-
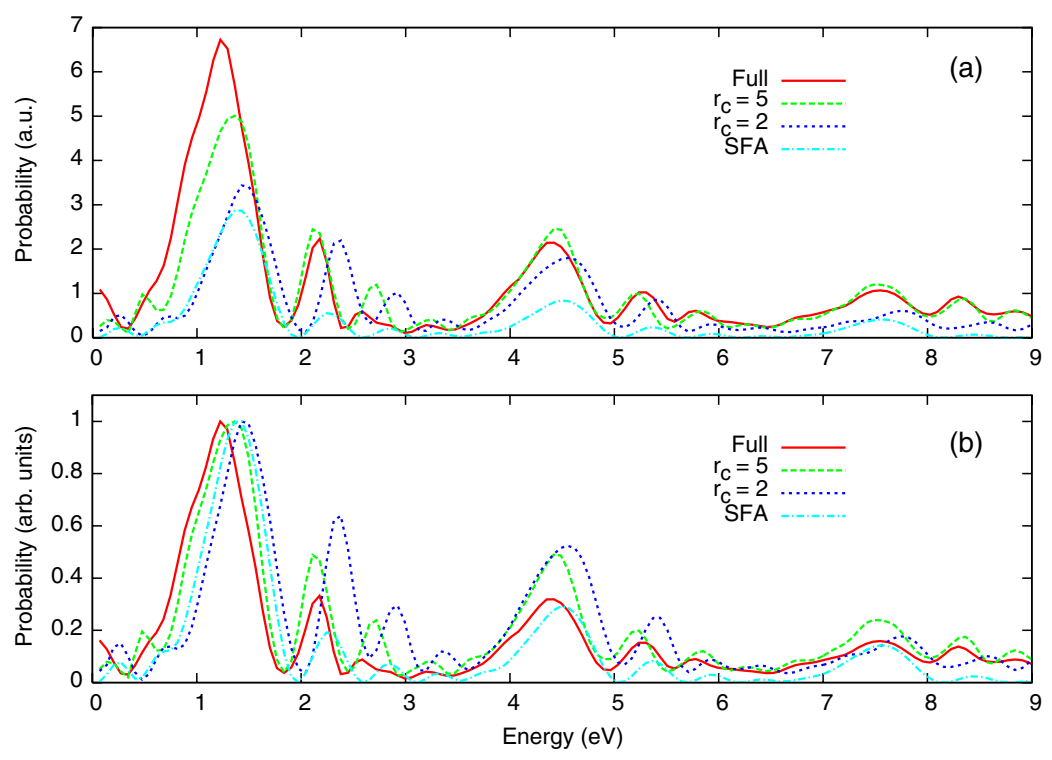

FIG. 5. (Color online) (a) Absolute electron energy spectra of Fig. 2. (b) Same spectra but normalized at the first peak. 

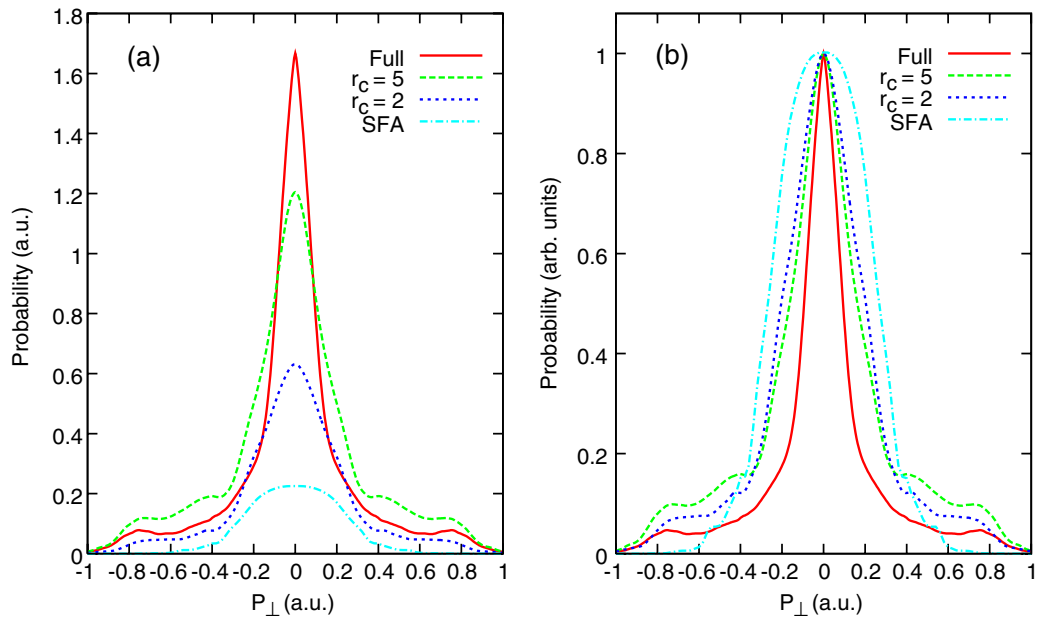

FIG. 6. (Color online) (a) Perpendicular momentum distribution from the spectra of Fig. 2. (b) Same spectra but normalized at the peak.

tribution is presented by normalizing it at the peak position, then the broadening of successive models can be easily seen, as shown in Fig. 6(b). The narrowing of the transverse momentum spectra due to Coulomb focusing has been treated previously using the quasiclassical trajectory method including tunneling [38].

\section{E. Angular momentum analysis of ATI electrons}

The 2D momentum spectra or the angular distributions of the ATI electrons presented in Fig. 3 above can be analyzed by examining the angular momentum compositions of the ATI electrons. In Table I, we compare the weights (the largest one is normalized to 1 ) of the $L$ distributions for electrons near the first ATI peak, at $p=0.17$, from the three TDSE calculations. For the exact calculation, $L=5$ is the dominant one; thus, there are six peaks in Fig. 3(a). For the $r_{c}=5$ and 2 cutoff potential calculations, the dominant angular momentum is $L=3$, and thus there are four peaks. In the exact calculation, the radial stripes in Fig. 3(a) require that $L=5$ should be the dominant angular momentum for electron momentum up to $p=0.2$ and the calculation does show that is indeed the case. For Figs. 3(b) and 3(c), the stripes are nearly vertical since at lower energies, the dominant angular momentum of the electron changes to $L=1$ from $L=3$ to make the stripes at lower energies appear vertical. Note that in the SFA shown in Fig. 4(d), the low-energy part is dominated by two vertical stripes, indicating that $L=1$ is the dominant angular momentum. Similarly, for the 400-nm case shown in Fig. 4 , the exact calculation has maximum weight at $L=3$,

TABLE I. The relative weights of the five largest angular momentum components of the electron with momentum $p=0.17$ for the case of Fig. 3.

\begin{tabular}{cccccc}
\hline \hline \multirow{2}{*}{ Full potential } & $L=5$ & $L=3$ & $L=1$ & $L=7$ & $L=4$ \\
& 1.00 & 0.23 & 0.17 & 0.05 & 0.02 \\
$r_{c}=5$ & $L=3$ & $L=1$ & $L=5$ & $L=2$ & $L=4$ \\
& 1.00 & 0.11 & 0.01 & 0.01 & 0.01 \\
$r_{c}=2$ & $L=3$ & $L=1$ & $L=5$ & $L=4$ & $L=2$ \\
& 1.00 & 0.18 & 0.02 & 0.01 & 0.01 \\
\hline \hline
\end{tabular}

while with the cutoff potentials the maximum weight is at $L=1$.

We have found that the radial stripes are ubiquitous features of the low-energy electron momentum spectra for atoms. Besides Figs. 2(a) and 3(a), we have performed many more TDSE calculations and six more examples are shown in Fig. 7, with the parameters given in the figure captions. In all cases the radial stripes are clearly seen, except for the 800-nm case in Fig. 7(e), which is somewhat distorted. These radial stripes also appeared in the calculations of Arbó et al. [13] (which have been confirmed by us). By analyzing the angular momentum distributions of these electron spectra in the low-energy region, together with additional calculations that are not shown here, we summarized the dominant $L$ values from these calculations in Table II. The dominant $L$ and the minimum number of photons, $N$, needed to ionize the atom for each case are also shown.

\section{F. Model of predicting the dominant angular momentum of the photoelectron at low energies}

To understand the value of the dominant $L$, we use a model based on the multiphoton absorption for the ATI spectra at low energies. From each initial state with angular momentum $l$, absorption of one photon takes the electron to $L$ $=l-1$ or $L=l+1$. Absorption of one more photon from each one of these levels will populate $L=l-2, L=l$ and $L=l, L$ $=l+2$, respectively, except when $L=0$ is reached. In the latter case, only a transition from $L=0$ to $L=1$ is possible. By assuming equal probability for making transitions from $L$ to $L-1$ and from $L$ to $L+1$, we found that the predicted maximum weight in $L$ for absorbing a given number of photons is smaller than what was found in Table II. Since the dipole transition matrix element from $L$ to $L+1$ in general is larger than for the transition from $L$ to $L-1$, we adjusted the relative probability and found that a ratio of 1.335:0.665 for $N$ $<13$ and 1.285:0.715 for $N \geqslant 13$ would predict the correct $L$ shown in Table II. In this model, the dominant $L$ for the low-energy ATI electron depends only on the number of photons, $N$, absorbed. In Fig. 8 we show the values of the dominant $L$ versus the minimum number of photons needed to ionize the atom for ionization from initial $s$ and $p$ orbitals. 

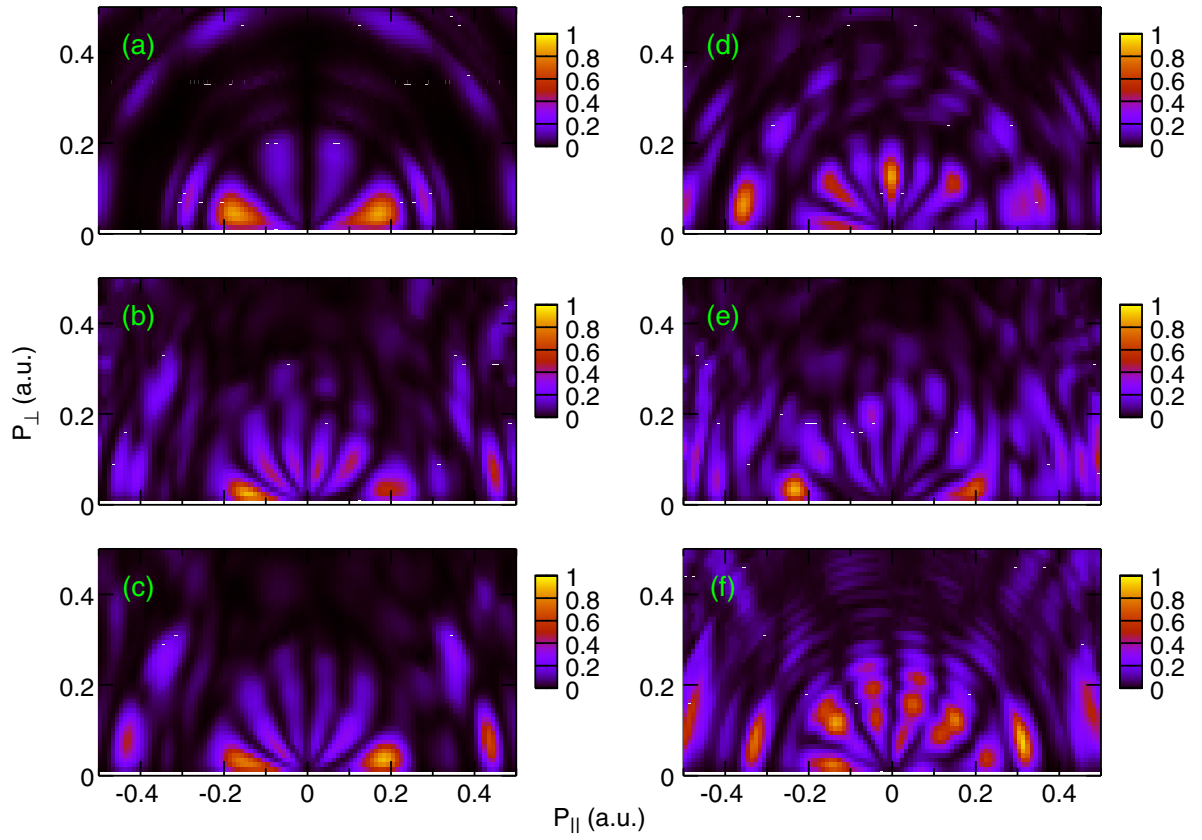

FIG. 7. (Color online) Photoelectron 2D momentum distributions for single ionization by a 10-fs (FWHM) laser pulse. (a) Ar, with a wavelength of $400 \mathrm{~nm}$ at peak intensity of $1.7 \times 10^{14} \mathrm{~W} / \mathrm{cm}^{2}$; (b) Ar, with a wavelength of $590 \mathrm{~nm}$ at peak intensity of $2.8 \times 10^{14} \mathrm{~W} / \mathrm{cm}^{2}$; (c) $\mathrm{Ne}$, with a wavelength of $600 \mathrm{~nm}$ at peak intensity of $2.1 \times 10^{14} \mathrm{~W} / \mathrm{cm}^{2}$; (d) $\mathrm{H}$, with a wavelength of $800 \mathrm{~nm}$ at peak intensity of $0.8 \times 10^{14} \mathrm{~W} / \mathrm{cm}^{2}$; (e) $\mathrm{H}$, with a wavelength of $800 \mathrm{~nm}$ at peak intensity of $2.03 \times 10^{14} \mathrm{~W} / \mathrm{cm}^{2}$; (f) He, with a wavelength of $400 \mathrm{~nm}$ at peak intensity of $6.2 \times 10^{14} \mathrm{~W} / \mathrm{cm}^{2}$.
According to this model, the dominant $L$, or the number of radial stripes in the $2 \mathrm{D}$ spectra, does not change with laser intensity until the next channel closing. Thus the radial stripes would survive the volume integration and these features are the most pronounced structures in the 2D spectra in Maharjan et al. [7]. At present we do not have a theoretical interpretation of the empirical dominant $L$ presented here.

TABLE II. The relation of the dominant angular momentum $L$ with the minimum number of photons, $N$, needed to ionize the atom. $L$ was found from the TDSE calculations. The laser pulse is $10 \mathrm{fs}$ (FWHM). Targets are Ar, Ne, H, and He. Laser wavelength $\lambda$, peak intensity $I_{0}$, and the Keldysh parameter $\gamma$ are listed together with $N$ and $L$.

\begin{tabular}{lccccc}
\hline \hline Target & $\lambda(\mathrm{nm})$ & $I_{0}\left(10^{14} \mathrm{~W} / \mathrm{cm}^{2}\right)$ & $\gamma$ & $N$ & $L$ \\
\hline $\mathrm{Ar}$ & 400 & 1.700 & 1.76 & 6 & 3 \\
$\mathrm{Ar}$ & 400 & 3.200 & 1.16 & 7 & 4 \\
$\mathrm{Ar}$ & 590 & 0.900 & 1.64 & 9 & 4 \\
$\mathrm{Ar}$ & 590 & 1.600 & 1.23 & 10 & 5 \\
$\mathrm{Ar}$ & 590 & 2.100 & 1.07 & 11 & 6 \\
$\mathrm{Ar}$ & 590 & 2.800 & 0.93 & 12 & 5 \\
$\mathrm{Ar}$ & 600 & 1.400 & 1.29 & 10 & 5 \\
$\mathrm{Ne}$ & 600 & 2.100 & 1.24 & 14 & 5 \\
$\mathrm{H}$ & 800 & 0.800 & 1.19 & 12 & 6 \\
$\mathrm{H}$ & 800 & 2.030 & 0.75 & 17 & 7 \\
$\mathrm{H}$ & 910 & 0.494 & 1.34 & 13 & 5 \\
$\mathrm{H}$ & 910 & 0.972 & 0.95 & 16 & 6 \\
$\mathrm{H}$ & 910 & 1.946 & 0.67 & 22 & 8 \\
$\mathrm{He}$ & 400 & 4.100 & 1.42 & 10 & 4 \\
$\mathrm{He}$ & 400 & 6.200 & 1.15 & 11 & 5 \\
\hline \hline
\end{tabular}

\section{G. Electron momentum spectra from the modified SFA model}

The MFSA has been shown to give an accurate ionization rate for $\mathrm{He}^{+}$by a 400-nm laser pulse by Chirilă and Potviege [27]. Here we apply the same model to calculate the 2D momentum spectra and the electron energy distributions for Ar atoms ionized by a laser pulse of wavelength $400 \mathrm{~nm}$, peak intensity $3.2 \times 10^{14} \mathrm{~W} / \mathrm{cm}^{2}$, and pulse duration $10 \mathrm{fs}$. The calculated total ionization probability from the MSFA is 3.22, which is 26 times larger than the SFA and 6 times larger than the TDSE. For the electron energy spectra and the 2D momentum spectra, as shown in Fig. 9, there is little difference between the MSFA and SFA. Since the MSFA does not give the correct total ionization probability either for the present case or for other cases we have tried, we conclude that the MSFA as given by Eq. (18) is not a useful

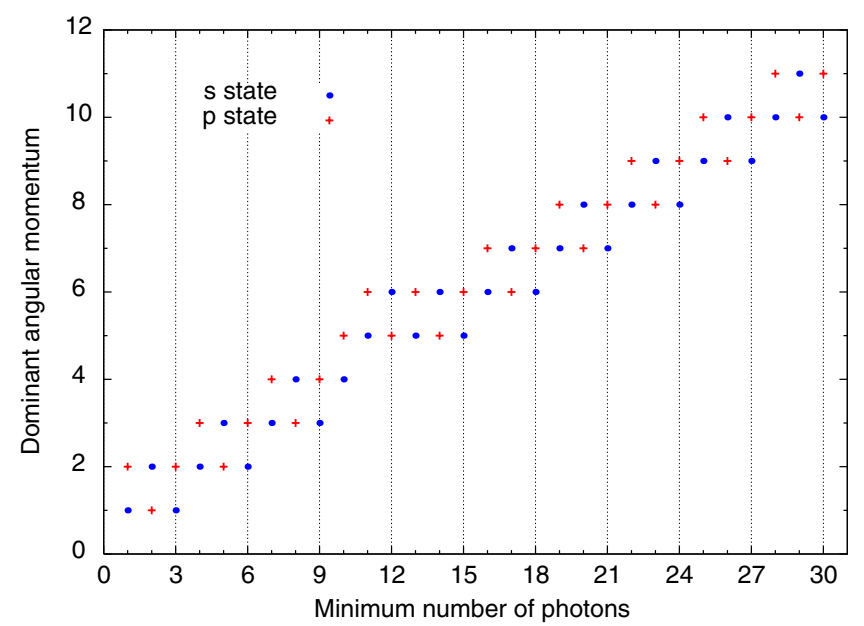

FIG. 8. (Color online) Dependence of the dominant $L$ on the minimum number $N$ of photons needed to ionize the atom for atoms initially in the $s$ or $p$ state. 

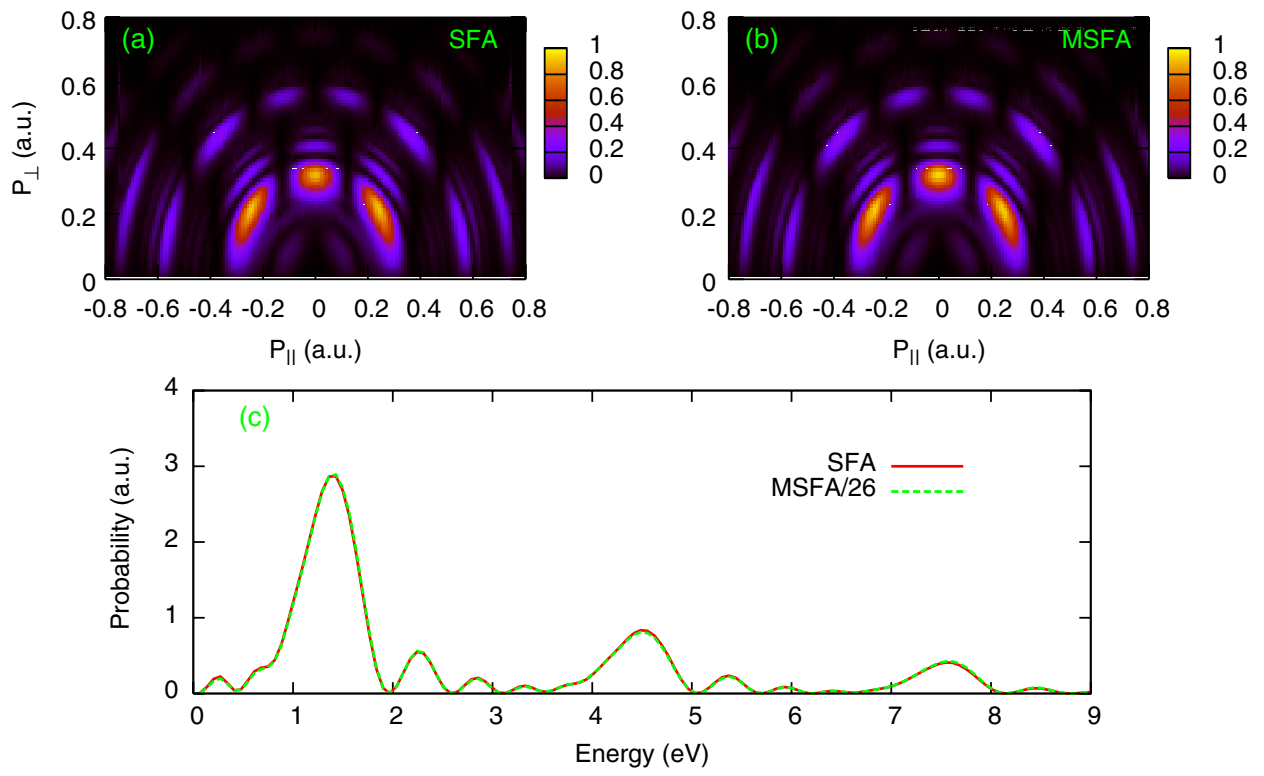

FIG. 9. (Color online) Photoelectron spectra for single ionization of argon by a 10 fs (FWHM) laser pulse with the wavelength of $400 \mathrm{~nm}$ at the peak intensity of $3.2 \times 10^{14} \mathrm{~W} / \mathrm{cm}^{2}$. (a) $2 \mathrm{D}$ momentum distribution calculated by SFA, (b) 2D momentum distribution calculated by MSFA, and (c) the SFA and MSFA energy distribution.

alternative simple theory beyond the SFA for treating laseratom or laser-molecule interactions.

\section{H. Electron momentum spectra from the orthonormalized SFA model}

In this subsection we check if the SFA is improved by requiring that the final state (the Volkov state) be orthonormal to the initial state. Figure 10 shows the electron energy spectra from the OSFA and compares it to the spectra from the SFA and TDSE. The target is atomic hydrogen and the laser is $10 \mathrm{fs}$, with peak intensities of (a) $1.0 \times 10^{14} \mathrm{~W} / \mathrm{cm}^{2}$ and (b) $2.0 \times 10^{14} \mathrm{~W} / \mathrm{cm}^{2}$. In (a), the total ionization probability from the OSFA is 2.8 times larger than from the TDSE, while the SFA is 11 times smaller than the TDSE. In (b), the OSFA is 1.28 times larger than the TDSE, while the SFA is 8.5 times smaller than the TDSE. Thus it appears that the OSFA gives a good improvement over the SFA in the total ionization probability. Unfortunately, when applied to other systems like $\mathrm{He}^{+}$and Ar, we did not find such improvement. In the meanwhile, Fig. 10 shows that the electron spectra from the OSFA agree better with the SFA. In other words, the simple OSFA cannot be taken as an improvement over the SFA in general either. We comment that the present OSFA requires that the final Volkov state function be orthonormal to the initial state. In a recent paper, Gordon et al. [39] emphasized the role of many-electron dynamics in highharmonic generation. Their model is equivalent to requiring that the final Volkov state be orthogonal to all occupied states, but excluding the initial orbital. If orthogonality of the Volkov state is important, it is not clear why orthogonality with respect to the initial occupied state is not needed. We comment that the equivalent of their theory for ATI electrons would not modify the SFA much at all.

Based on the results of the OSFA and MSFA, we conclude that both models do not improve the SFA for treating laseratom interactions. While a simple model for laser-atom interactions is not essential since the TDSE can be used routinely, the lack of such a model is problematic for laser- molecule interactions where solving the TDSE for molecules, even within the SAE model, is far from efficient at present and in the foreseeable future.

\section{SUMMARY}

In this paper we investigated the two-dimensional electron momentum spectra of the single ionization of atoms by intense laser pulses by solving the time-dependent Schrödinger equation for different laser intensities and wave-

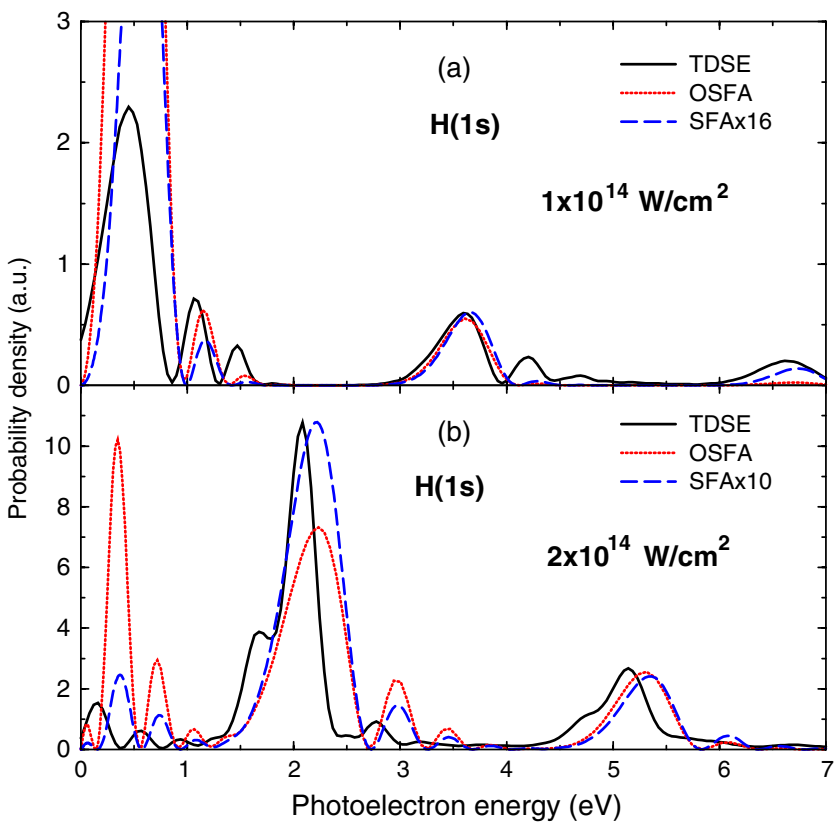

FIG. 10. (Color online) Comparison of photoelectron spectra of $\mathrm{H}(1 s)$ by a 10-fs (FWHM) laser pulse with wavelength of $400 \mathrm{~nm}$ at peak intensity of (a) $1.0 \times 10^{14} \mathrm{~W} / \mathrm{cm}^{2}$ and (b) $2.0 \times 10^{14} \mathrm{~W} / \mathrm{cm}^{2}$, calculated by different theoretical models. Note that the results from the SFA have been normalized, as shown on the labels. See text. 
lengths. Comparing to the results from the strong-field approximation, we noticed very important difference in the electron spectra for small momenta. As shown in Fig. 3, the TDSE results show pronounced islands of peaks fanning out radially, while with the removal of the long-range Coulomb potential in the TDSE, as well as in the SFA calculations, these fans begin to deform and become stripes that are nearly perpendicular to the polarization axis. These fans are related to the fact that at low energies the ATI electrons usually have one dominant orbital angular momentum only. This dominant angular momentum appears to be related to the minimum number of photons needed to ionize the atom at that intensity and wavelength. We have found an empirical rule to determine this dominant angular momentum and thus the number of peaks fanning out in the two-dimensional momentum spectra. Note that these peaks are the main features of the low-energy spectra and they do not change much until higher intensity which would require one more photon to ionize the atom. When that happens, the dominant angular momentum would increase or decrease by one unit and new islands will be fanning out with distinct different parities for $p_{\|}=0$. These islands of peaks fanning out from the center are the major features in the experimental 2D electron momentum spectra [6,7]. We note that the experimental spectra usually resulting from integrating a range of laser intensities and thus only features that change slowly can survive the volume integration. In agreement with Arbó et al. these low-energy electron structures are not due to rescattering, but rather by the long-range Coulomb potential effect, as confirmed by the disappearance of these fans when the Coulomb potential tail is removed.

We comment that the important role of the Coulomb potential played in the low-energy electron momentum spectra does not translate into the electron energy spectra. Except for the slight shift in the energy spectra towards lower energies, there is no clearly observable difference in the TDSE results and SFA results. For the projected momentum spectra in the perpendicular direction, sharper distributions from the TDSE are observed as the result of "Coulomb focusing". In earlier experiments, the electron momentum in the parallel directions has been measured. A major effort has been made to "understand" whether the distribution near $p_{\|}=0$ is a dip or a maximum. Based on the 2D momentum spectra discussed in this paper, we expect that the structure of peak or dip at $p_{\|}$ $=0$ depends on the laser intensity and the wavelength, and no general significance can be assigned.

Finally we emphasize that the fanlike radial stripes are ubiquitous features of low-energy 2D electron momentum spectra from atoms for lasers of different wavelengths and intensities. While we have identified an empirical rule for figuring out the dominant angular momentum of the lowenergy electrons for atoms, we have not been able to provide a more satisfactory explanation of its origin.

\section{ACKNOWLEDGMENTS}

This work was supported in part by Chemical Sciences, Geosciences and Biosciences Division, Office of Basic Energy Sciences, Office of Science, U.S. Department of Energy. T.M. is also supported by financial aid from the research fund of the University of Electro-communications, the 21st century COE program on "Coherent Optical Science" and the Ministry of Education, Culture, Sports, Science, and Technology, Japan.
[1] M. Wickenhauser, X. M. Tong, and C. D. Lin, Phys. Rev. A 73, 011401(R) (2006).

[2] M. Wickenhauser, X. M. Tong, D. C. Arbó, J. Burgdörfer, and C. D. Lin, Phys. Rev. A 74, 041402(R) (2006).

[3] J. N. Bardsley, A. Szoke, and M. J. Comella J. Phys. B 21, 3899 (1988).

[4] R. R. Freeman, P. H. Bucksbaum, H. Milchberg, S. Darack, D. Schumacher, and M. E. Geusic, Phys. Rev. Lett. 59, 1092 (1987).

[5] R. Wiehle, B. Witzel, H. Helm, and E. Cormier, Phys. Rev. A 67, 063405 (2003).

[6] A. Rudenko, K. Zrost, C. D. Schröter, V. L. B. de Jesus, B. Feuerstein, R. Moshammer, and J. Ullrich, J. Phys. B 37, L407 (2004).

[7] C. M. Maharjan, A. S. Alnaser, I. Litvinyuk, P. Ranitovic, and C. L. Cocke, J. Phys. B 39, 1955 (2006).

[8] J. L. Krause, K. J. Schafer, and K. C. Kulander, Phys. Rev. A 45, 4998 (1992).

[9] X. M. Tong and S. I. Chu, Phys. Rev. A 55, 3406 (1997).

[10] X. M. Tong and Shih-I Chu, Chem. Phys. 217, 119 (1997).

[11] C. J. Joachain, M. Dorr, and N. Kylstra, Adv. At., Mol., Opt. Phys. 42, 225 (1999).

[12] S. Chelkowski, A. D. Bandrauk, and A. Apolonski, Phys. Rev.
A 70, 013815 (2004).

[13] D. C. Arbó, S. Yoshida, E. Persson, K. I. Dimitriou, and J. Burgdöorfer, Phys. Rev. Lett. 96, 143003 (2006).

[14] F. H. M. Faisal and G. Schlegel, J. Mod. Opt. 53, 207 (2006).

[15] L. V. Keldysh, Sov. Phys. JETP 20, 1307 (1964).

[16] F. H. M. Faizal, J. Phys. B 6, L89 (1973).

[17] H. R. Reiss, Phys. Rev. A 22, 1786 (1980).

[18] X. M. Tong and C. D. Lin, J. Phys. B 38, 2593 (2005).

[19] D. O. Harris, G. G. Engerholm, and W. D. Gwinn, J. Chem. Phys. 43, 1515 (1965).

[20] A. S. Dickinson and P. R. Certain, J. Chem. Phys. 49, 4209 (1968).

[21] J. C. Light and R. B. Walker, J. Chem. Phys. 65, 4272 (1976).

[22] M. Lewenstein, Ph. Balcou, M. Yu. Ivanov, Anne L'Huillier, and P. B. Corkum, Phys. Rev. A 49, 2117 (1994).

[23] R. Shakeshaft, R. M. Potvliege, M. Dörr, and W. E. Cooke, Phys. Rev. A 42, 1656 (1994).

[24] G. F. Gribakin and M. Yu. Kuchiev, Phys. Rev. A 55, 3760 (1997).

[25] V. P. Krainov and B. Shokri, JETP 80, 657 (1995).

[26] V. P. Krainov, J. Opt. Soc. Am. B 14, 425 (1997).

[27] C. C. Chirilă and R. M. Potvliege, Phys. Rev. A 71, 021402(R) (2005). 
[28] B. Bransden and M. R. C. McDowell, Charge Exchange and the Theory of Ion-Atom Collisions (Oxford University Press, Oxford, 1992).

[29] H. C. Brinkman and H. A. Kramers, Proc. R. Acad. Sci. Amsterdam 33, 973 (1930).

[30] J. R. Oppenheimer, Phys. Rev. 31, 349 (1928).

[31] C. D. Lin, S. C. Soong, and L. N. Tunnell, Phys. Rev. A 17, 1646 (1978).

[32] D. Bauer, D. B. Milošević, and W. Becker, Phys. Rev. A 72, 023415 (2005).

[33] D. Bauer, D. B. Milošević, and W. Becker, J. Mod. Opt. 53, 135 (2006)

[34] D. B. Milošević, G. G. Paulus, D. Bauer, and W. Becker, J.
Phys. B 39, R203 (2006).

[35] X. M. Tong, Z. X. Zhao, and C. D. Lin, Phys. Rev. A 66, 033402 (2002).

[36] D. Comtois, D. Zeidler, H. Pépin, J. C. Kieffer, D. M. Villeneuve, and P. B. Corkum, J. Phys. B 38, 1923 (2005).

[37] A. Rudenko, K. Zrost, Th. Ergler, A. B. Voitkiv, B. Najjari, V. L. B. de Jesus, B. Feuerstein, C. D. Schröter, R. Moshammer, and J. Ullrich, J. Phys. B 38, L191 (2005).

[38] K. I. Dimitriou, D. G. Arbó, S. Yoshida, E. Persson, and J. Burgdörfer, Phys. Rev. A 70, 061401(R) (2004).

[39] A. Gordon, F. Kartner, N. Rohringer, and R. Santra, Phys. Rev. Lett. 96, 223902 (2006). 